\title{
Amplified Spontaneous Emission in Short-Pulse Excimer Amplifiers
}

\author{
G. Kühnle, U. Teubner, and S. Szatmári` \\ Max-Planck-Institut für biophysikalische Chemie, Abteilung Laserphysik, \\ D-3400 Göttingen, Fed. Rep. Germany
}

Received 15 February 1990/Accepted 22 February 1990

\begin{abstract}
We have found a simple analytical expression which describes the relation between amplified spontaneous emission (ASE) and small-signal gain in short-pulse amplifiers. It is also shown that the contrast of the short pulse to the ASE is weakly dependent on the saturation of the ASE, and influenced mainly by the saturation of the short pulse. The theoretical considerations were verified by measurements.
\end{abstract}

PACS: $42.60,42.55$

Amplification of short pulses always demands a tradeoff between good extraction efficiency, high gain and low ASE level. Especially in short-pulse excimer lasers ASE plays an important role [1-6], due to the short wavelength, the large emission cross section of excimers and because of the lack of good saturable absorbers in the UV region [7]. The ASE not only contributes to the output and thus decreases the contrast ratio, but it depletes the gain significantly, leading to much reduced gain seen by the short pulse. In this paper considerations are presented to avoid these effects.

\section{Theoretical Model}

For the estimation of the ASE and the depleted gain we developed a model assuming a reduced four level laser system for the energetic states of the laser molecules and a steady state condition. This seems reasonable since the ASE pulse duration is much longer than the lifetime of the upper laser level. Using these assumptions one gets two equations for the ASE-intensity

* Permanent address: Research Group on Laser Physics of the Hungarian Academy of Sciences, JATE University, Dóm tér 9, H-6720 Szeged, Hungary travelling to the right side $\left(I_{+}\right)$and to the left side $\left(I_{-}\right)$ [8-13]:

$$
\begin{aligned}
d \hat{I}_{ \pm} & = \pm g\left(a+\hat{I}_{ \pm}\right) d z, \\
g & =\frac{g_{0}}{1+\left(\hat{I}_{+}+\hat{I}_{-}\right) /\left(1+R \tau_{\text {tot }}\right)},
\end{aligned}
$$

where

$$
\begin{array}{lll}
a=\frac{\tau_{\text {tot }}}{\tau_{\text {rad }}} \frac{\Delta v_{\mathrm{ASE}}}{\Delta v_{\mathrm{Gain}}} \frac{\delta \Omega}{4 \pi}, & g_{0}=\sigma^{*} N_{0}^{*}, & g=\sigma^{*} N^{*}, \\
N_{0}^{*}=N_{\text {tot }} \frac{R \tau_{\text {tot }}}{1+R \tau_{\text {tot }}}, & \hat{I}_{ \pm}=I_{ \pm} / I_{s}, & I_{s}=\frac{h \nu}{\sigma^{*} \tau_{\text {tot }}}
\end{array}
$$

and the symbols have the following meanings:

$\tau_{\text {tot }}:$ total lifetime of the upper laser level including non-radiative processes;

$\tau_{\text {rad }}:$ radiative lifetime of the upper laser level;

$\Delta v_{\text {Gain }}$ : bandwidth of the gain;

$\Delta v_{\mathrm{ASE}}$ : bandwidth of the ASE;

$\delta \Omega$ : average solid angle which is seen by the ASE;

$R$ : pumping rate;

$I_{\mathrm{s}}$ : saturation intensity;

$h$ : Planck's constant;

$v$ : laser frequency;

$\sigma^{*}$ : excited state cross section for stimulated emission to the ground state;

$N_{\text {tot }}$ : total number of excitable molecules per volume; 
$N_{0}^{*}$ : number of excited molecules per volume without gain depletion by ASE;

$N^{*}$ : number of excited molecules per volume including gain depletion by ASE;

$g_{0}$ : small-signal gain coefficient;

$\mathrm{g}$ : depleted small-signal gain coefficient;

$a$ : a constant which describes the temporal, spectral, and spatial behaviour of the ASE.

Equations (1a) and (1b) are the basic equations used in many calculations (see for example [8]), but generally they have been solved numerically or only approximately. We found that the integral of these equations can be obtained in an explicit form:

$$
\begin{aligned}
& \frac{2 a+\hat{I}_{\mathrm{in}^{+}}+\hat{I}_{\mathrm{in}}}{1+R \tau_{\text {tot }}}[\exp (\langle g\rangle L)-1] \\
& \quad+\left(1-\frac{2 a}{1+R \tau_{\text {tot }}}\right)\langle g\rangle L=\left\langle g_{0}\right\rangle L
\end{aligned}
$$

with $\left\langle g_{0}\right\rangle L=\int_{0}^{L} g_{0} d z$ and

$\ln \left(\frac{\hat{I}_{\text {out }^{+}+a}+a}{\hat{I}_{\text {in }^{+}}+a}\right)=\ln \left(\frac{\hat{I}_{\text {out }^{-}+a}}{\hat{I}_{\text {in }^{-}}+a}\right)=\langle g\rangle L=\int_{0}^{L} g \cdot d z$

$\hat{I}_{\text {in }^{+}}:$normalized input intensity on the left side;

$\hat{I}_{\text {in }^{-}}:$normalized input intensity on the right side;

$\hat{I}_{\text {out }^{+}}$: normalized output intensity on the right side;

$\hat{I}_{\text {out }^{-}}$: normalized output intensity on the left side;

$\left\langle g_{0}\right\rangle$ : average small-signal gain coefficient;

$\langle g\rangle$ : average depleted small-signal gain coefficient.

Taking into account that $\hat{I}_{\mathrm{in}^{+}}=\hat{I}_{\mathrm{in}}=0$ and $2 a \ll 1+R \tau_{\text {tot }}$ and assuming a rectangular pulse form for $g_{0}$ with a width of $\tau_{\text {Gain }}$ one gets:

$\frac{2 a}{1+R \tau_{\text {tot }}}[\exp (\langle g\rangle L)-1]+\langle g\rangle L=\left\langle g_{0}\right\rangle L$

and

$E_{\mathrm{ASE}}=a \tau_{\mathrm{Gain}} I_{\mathrm{s}} A[\exp (\langle\mathrm{g}\rangle L)-1]$,

where $A$ is the cross-sectional area of the ASE.

From (3) one can calculate the average depleted gain $\langle g\rangle$ and the ASE output energy $E_{\mathrm{ASE}}$. The inversion is not significantly depleted by ASE if $2 a \exp \left(\left\langle g_{0}\right\rangle L\right) \ll 1$ is fulfilled [as can be obtained from (1)]. Then for a desired small signal gain $\left[\exp \left(\left\langle g_{0}\right\rangle L\right)\right]$ the average solid angle seen by the ASE has to be chosen as:

$\delta \Omega \ll 4 \pi \frac{\tau_{\text {rad }}}{\tau_{\text {tot }}} \frac{\Delta v_{\text {Gain }}}{\Delta v_{\text {ASE }}} \frac{1}{2} \exp \left(-\left\langle g_{0}\right\rangle L\right)$.

Using (4) one can design an amplifier properly to avoid gain depletion (GD) by ASE.

For the description of the short pulse amplification the following assumptions were made. A long pulse sees an inversion which is stabilized by the ASE to the depleted value. Therefore the small signal gain coefficient seen by the ASE $\left(g_{\text {ASE }}\right)$ is equal to the depleted small signal gain coefficient $(g)$. Due to the limited relaxation times in the excited states [14-17], only a fraction of the inverted molecules are available for the short pulse, which then sees a somewhat lower inversion than a long pulse would see. It is reasonable to write:

$g_{\text {short }}=K g=K g_{\text {ASE }}$

$g_{\text {short }}:$ short pulse small signal gain coefficient;

$K$ : constant which describes the accessable inversion for the short pulse $(1 \geqq K \geqq 0)$.

The value of $K$ is dependent on the laser medium and may vary over a large range for different laser media.

Assuming a rectangular short pulse one can calculate the amplification $\left(G_{\text {short }}\right)$ by the slightly generalized Frantz-Nodvik-Formula [18]:

$G_{\text {short }}=\frac{1}{\hat{E}_{\text {in }}} \ln \left[1+\exp \left(\left\langle g_{\text {short }}\right\rangle L\right)\left(\exp \left(\hat{E}_{\text {in }}\right)-1\right)\right]$,

where $\left\langle g_{\text {short }}\right\rangle L=\int_{0}^{L} g_{\text {short }} d z, \hat{E}_{\mathrm{in}}=E_{\mathrm{in}} / E_{\mathrm{s}}, E_{\mathrm{s}}=\frac{h v}{\sigma^{*}}$, and $E_{\text {in }}$ is the short pulse input energy density.

Using (3), (5), and (6) one can calculate the short pulse gain which is the result of GD by ASE and saturated amplification of the short pulse.

Assuming no saturation (i.e. $\left.\widehat{E}_{\text {in }} \ll \exp \left[-\left\langle g_{\text {short }}\right\rangle L\right]\right)$, Eqs. (3), (5), and (6) can be combined as:

$G_{\text {short }}=\left(1+\frac{E_{\text {ASE }}}{a \tau_{\text {Gain }} I_{s} A}\right)^{K}$.

The importance of the relationship expressed by (7) is that it can be used as a simple method to determine the depleted small signal gain for the short pulse by measuring only the ASE energy for amplifiers characterized by a known value of $K$. When this value is not known, (7) provides a simple way to determine $K$, by measuring the dependence of $G_{\text {short }}$ on $E_{\mathrm{ASE}}$. Formerly $K$ could only be estimated by complicated gain dynamics measurements [14-17].

An additional even more interesting fact can also be seen from (7). If the short pulse saw the same inversion as a long pulse $(K=1)$ the ratio of the smallsignal gain of the short pulse and the ASE output energy would be approximately constant. This means that, regardless of whether the ASE is already in saturation or not, the contrast between short pulse and ASE would always be constant. To get a high contrast one has to choose a laser medium with a large value of $K$, which can be estimated from former gain dynamics 
measurements $[14,15,17]$. For $\mathrm{KrF}^{*}, K$ is expected to be the largest, therefore the contrast is only weakly dependent on the ASE.

The contrast is strongly decreased when the short pulse is in saturation as can be seen from (6). On the other hand, saturation of the short pulse at least in the last amplifier is necessary for good extraction efficiency, since the final short-pulse energy for a given amplifier medium is determined by the cross section of the laser beam and by the degree of saturation (by the ratio of the output energy density and the saturation energy density). In preamplifiers, where efficiency is not a key point, saturation only decreases the contrast without increasing significantly the short pulse energy after the last amplifier. For this reason saturation in preamplifiers should be avoided.

\section{Experimental}

For the experimental study of GD by ASE and to verify Eq. (7), measurements were performed on the amplifier section of a commercial Lambda Physik EMG150 ES excimer laser. This discharge section has a $2.5 \mathrm{~cm}$ $\times 1 \mathrm{~cm}$ cross section and a discharge length of $84 \mathrm{~cm}$. It was filled with the standard gas fill for $\mathrm{KrF}$ operation (6 mbar $\mathrm{F}_{2}, 150$ mbar $\mathrm{Kr}, 1.65 \mathrm{bar} \mathrm{He}$ ) and operated with a charging voltage of $30 \mathrm{kV}$. We found that the electric energy pumped into the cell - optimized for long pulse operation - is far too high when amplification of short pulses is needed. For this reason we removed a quarter of the capacitors, both at the capacitor bank and at the laser tube. All the measurements in which the active length was changed by changing the number of preionization-pins (see below) were performed with this modified electric charging circuit.

The small signal gain of the excimer gain module was measured by a subpicosecond probe pulse. These pulses were generated by a short-pulse excimer-dyelaser system [19] delivering $500 \mathrm{fs}, 10 \mathrm{~mJ}$ pulses at $248 \mathrm{~nm}$. The pulses were attenuated by calibrated aluminum-coated filters to avoid saturation in the small-signal gain measurements. A $6 \mathrm{~mm}$ diameter aperture was used to select the middle, homogeneous part of the laser beam of originally $0.8 \mathrm{~cm} \times 2 \mathrm{~cm}$ cross section. This resulted in an probe-pulse energy density of approximately $1.8 \mathrm{~nJ} / \mathrm{cm}^{2}$.

The short-pulse laser system and the excimer amplifier were synchronized by two EMG 97 Lambda Physik active synchronization control units. The short pulse was adjusted to be in the middle of the Gaussianlike $15 \mathrm{~ns}$ long ASE pulse. The overall jitter between the short pulse and the ASE pulse was approximately $3 \mathrm{~ns}$, which resulted in a negligible shot-to-shot fluctuation of the gain for the short pulse.
The energy of the short pulse behind the excimer amplifier was measured some $8 \mathrm{~m}$ away (to reduce the ASE background) with a high voltage biplanar photodiode (Hamatsu R 1193U-02) and a fast oscilloscope (Tektronix 7104 with a 7A29 plug-in-amplifier), to distinguish between the short pulse and the long ASE pedestal.

For the measurement of the ASE energy $\left(E_{\mathrm{ASE}}\right)$ a Gentec ED 500 energy meter was put directly behind the output window of the amplifier, and the short input pulse was blocked. When $G_{\text {short }}$ and $E_{\text {ASE }}$ were measured as a function of the amplifier-length $(L)$ the length of the pumped volume was changed by disconnecting the necessary number of UV-preionizing electrodes starting from both ends. During these measurements the high voltage and therefore the break-down-voltage of the discharge channel were kept constant. This means that the current density in the active volume must be independent of the length of the discharge. Since the electrically stored energy is constant and the duration of the ASE pulse is found to be independent of length, part of the electrical energy does not contribute to the discharge, but is dissipated in a non-radiative process. This can lead to enhanced instabilities of the discharge with decreasing discharge length. This tendency has been observed experimentally, and that is why the shortest discharge length was limited to $24 \mathrm{~cm}$. As a result, the amplifier length was varied while the electrically excited molecules per volume and thus the non-depleted gain coefficient stayed constant.

\section{Experimental Results}

The experimental results are shown in Figs. 1-3. In Fig. 1 the dependence of the ASE energy is plotted as a function of the amplifier length. The ASE energy first rises rapidly and then only linearly with increasing length. In Fig. 2, $G_{\text {short }}$ is plotted on a logarithmic scale versus $L$. In the experimentally investigated range $G_{\text {short }}$ rises weakly with $L$. Figure 3 shows the measured

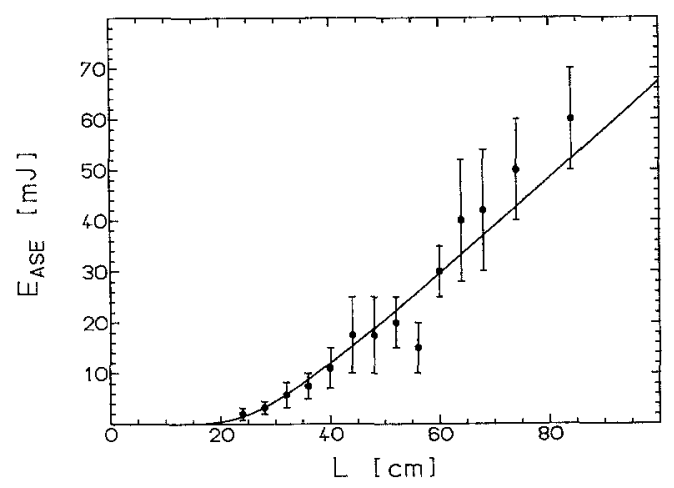

Fig. 1. ASE energy for different amplifier lengths. Points with error bars: experimental data, solid line: theoretical curve 


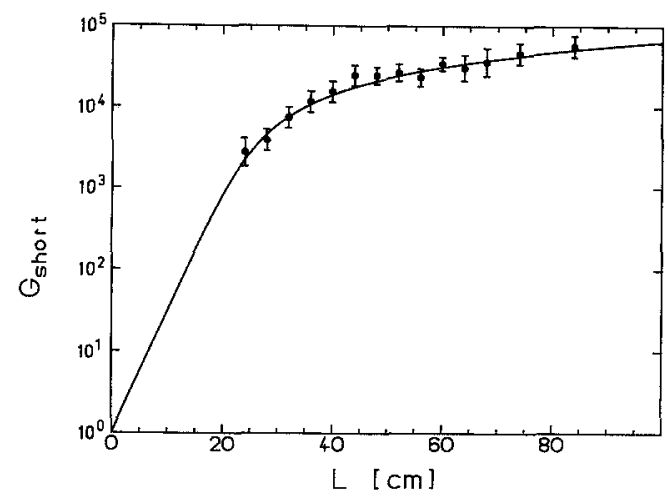

Fig. 2. Short-pulse small-signal gain for different amplifier lengths. Points with error bars: experimental data, solid line: theoretical curve

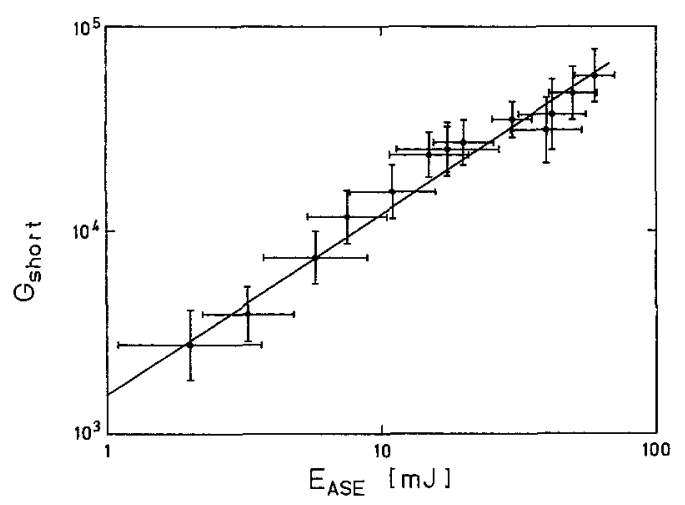

Fig. 3. Short pulse small signal gain versus ASE energy. Points with error bars: experimental data, solid line: theoretical curve

values of the logarithm of the short pulse small signal gain versus the logarithm of the ASE energy. It can be seen that the experimental data are along a straight line.

We have fitted (3) and (7) to the experimental data in Figs. 1 and 3. This resulted in the following parameters:

$$
\begin{aligned}
& K=0.9 \pm 0.3, \\
& \left\langle g_{0}\right\rangle=(0.38 \pm 0.08) \mathrm{cm}^{-1}, \\
& 2 a /\left(1+R \tau_{\text {tot }}\right)=(1 \pm 3) \times 10^{-4}, \\
& a \tau_{\mathrm{Gain}} I_{\mathrm{s}} A=(3 \pm 2) \times 10^{-4} \mathrm{~mJ} .
\end{aligned}
$$

The solid lines in Figs. 1-3 are theoretical curves with the above fit-parameters. All three curves show reasonable agreement with the experimental results.
As expected from gain dynamics measurements $[15,16]$ the value for $K$ is almost 1 . This means that the contrast is more or less constant independently of the ASE level.

\section{Conclusion}

A simple relationship is set up between the short-pulse small-signal gain and the ASE output energy. This shows proportionality between these two quantities for efficient short-pulse amplifiers like $\mathrm{KrF}^{*}$. Thus the contrast in these cases is practically independent of the ASE energy. The contrast deteriorates only when amplification of the short pulse is in saturation. The theoretical findings were supported by measurements.

Acknowledgements. The authors wish to thank F. P. Schäfer for his useful comments and critical reading of the manuscript. This work has been supported by the Bundesministerium für Forschung und Technologie.

\section{References}

1. J.H. Glownia, G. Arjavalingam, P.P. Sorokin, J.E. Rothenberg: Opt. Lett. 11, 79 (1986)

2. A.P. Schwarzenbach, T.S. Luk, I.A. McIntyre, U. Johann, A McPherson, K. Boyer, C.K. Rhodes: Opt. Lett. 11, 499 (1986)

3. S. Szatmári, F.P. Schäfer, E. Müller-Horsche, W. Mückenheim: Opt. Commun. 63, 305 (1987)

4. A. Endoh, M. Watanabe: Opt. Lett. 12, 906 (1987)

5. J.R.M. Barr, N.J. Everall, C.J. Hooker, I.N. Ross, M.J. Shaw, W.T. Toner: Opt. Commun. 66, 127 (1988)

6. J.P. Roberts, A.J. Taylor, P.H.Y. Lee, R.B. Gibson: Opt. Lett. 13, 734 (1988)

7. M. Watanabe, A. Endoh, N. Sarukura, S. Watanabe: J. Appl. Phys. 65, 428 (1989)

8. A.M. Hunter II, R.O. Hunter: IEEE J. QE-17, 1879 (1981)

9. D.D. Lowenthal, J.M. Eggleston: IEEE J. QE-22, 1165 (1986)

10. U. Ganiel, A. Hardy, G. Neumann, D. Treves: IEEE J. QE-11, 881 (1975)

11. A. Sasaki, K. Ueda, H. Takuma, K. Kasuya: J. Appl. Phys. 65, 231 (1989)

12. W.W. Rigrod: J. Appl. Phys. 34, 2602 (1963)

13. W.W. Rigrod: IEEE J. QE-14, 377 (1978)

14. P.B. Corkum, R.S. Taylor: IEEE J. QE-18, 1962 (1982)

15. S. Szatmári, F.P. Schäfer: J. Opt. Soc. Am. B 4, 1943 (1987)

16. A.J. Taylor, R.B. Gibson, J.P. Roberts: Appl. Phys. Lett. 52, 773 (1988)

17. Q. Zhao, S. Szatmári, F.P. Schäfer: Appl. Phys. B 47, 325 (1988)

18. L.M. Frantz, J.S. Nodvik: J. Appl. Phys. 34, 2346 (1963)

19. S. Szatmári, F.P. Schäfer: Opt. Commun. 68, 196 (1988) 